\title{
Intelligent Bio-Inspired Whale Optimization Algorithm for Color Image Based Segmentation
}

\author{
Athraa Jasim Mohammed* and Khalil Ibrahim Ghathwan \\ Department of Computer Science, University of Technology, Baghdad 00964, Iraq
}

\begin{abstract}
Color image segmentation is widely used methods for searching of homogeneous regions to classify them into various groups. Clustering is one technique that is used for this purpose. Clustering algorithms have drawbacks such as the finding of optimum centers within a cluster and the trapping in local optima. Even though inspired meta-heuristic algorithms have been adopted to enhance the clustering performance, some algorithms still need improvements. Whale optimization algorithm (WOA) is recognized to be enough competition with common meta-heuristic algorithms, where it has an ability to obtain a global optimal solution and avoid local optima. In this paper, a new method for color image based segmentation is proposed based on using whale optimization algorithm in clustering. The proposed method is called the whale color image based segmentation (WhCIbS). It was used to divide the color image into a predefined number of clusters. The input image in RGB color space was converted into L*a*b color space. Comparison of the proposed WhCIbS method was performed with the wolf color image based segmentation, cuckoo color image based segmentation, bat color image based segmentation, and k-means color image based segmentation over four benchmark color images. Experimental results demonstrated that the proposed WhCIbS had higher value of PSNR and lower value of RMSR in most cases compared to other methods.
\end{abstract}

ARTICLE INFO

Article history:

Received: 22 February 2020

Accepted: 27 May 2020

Published: 21 October 2020

DOI: https://doi.org/10.47836/pjst.28.4.14

E-mail addresses:

10872@uotechnology.edu.iq (Athraa Jasim Mohammed) 110039@uotechnology.edu.iq (Khalil Ibrahim Ghathwan)

*Corresponding author
Keywords: Clustering based technique, color image based segmentation, whale optimization algorithm

\section{INTRODUCTION}

Image segmentation is the separation of an image into homogeneous regions. It is frequently a main step in image investigation, item representation, visual 
image and numerous other image processing jobs. The aim of image segmentation is to represent image into different description to make it simpler and easier for analyzing (Zaitoun \& Aqel, 2015). There are four types of image: intensity image, binary image, indexed image, and color image (Gonzalez et al., 2003). Color image is classified into different color spaces such as RGB, $\mathrm{L}^{*} \mathrm{a} * \mathrm{~b}, \mathrm{YcbCr}$ and HSV.The $\mathrm{L}^{*} \mathrm{a} * \mathrm{~b}$ space is excessively used due to less execution time (Mathur \& Purohit, 2014). In this research, a color image based on RGB and L*a*b color spaces was applied.

In previous works, there are various segmentation techniques of image such as an edge or boundary based, threshold, clustering based, and soft computing approaches like genetic algorithm and Neural Network. Clustering based technique is an efficient technique (Dhanachandra et al., 2015). This study focused on clustering based technique.

Clustering can be defined as a process of splitting homogeneous objects in one cluster and heterogeneous objects in another cluster (Kapoor et al., 2017). In literature, there are different methods of clustering based such as: k-means clustering (Dhanachandra et al., 2015; Mathur \& Purohit, 2014), DBScan clustering method (Ye et al., 2003), subtractive clustering (Dhanachandra et al., 2015), and most recently used intelligent inspired optimization algorithms (i.e.meta-heuristic algorithms) in clustering like Wolf optimization algorithm (Kapoor et al., 2017), Cuckoo search algorithm (Nandy et al., 2015), Particle Swarm Optimization (Zhang et al., 2011), Bat optimization algorithm (Alagarsamy et al., 2019; Mishra \& Panda, 2018; Yang, 2010) and most recently introduced Whale Optimization algorithm (Mirjalili \& Lewis, 2016).

Whale Optimization algorithm (Mirjalili \& Lewis, 2016) is a nature inspired algorithm that uses in many fields and is proven successful (Mafarja \& Mirjalili, 2017; Thennarasu et al., 2020). In this paper, a new method for Color Image based Segmentation is proposed based on intelligent inspired Whale optimization algorithm, term as WhCIbS. The proposed method was compared with Wolf Color Image based Segmentation, termed as WCIbS, Cuckoo Color Image based Segmentation, termed as CCIbS, Bat Color Image based Segmentation, termed as BCIbS, and k-means Color Image based Segmentation, termed as $\mathrm{KmCIbS}$.

The organization of the remaining paper is as follows. Literature review includes related works and a brief description about image segmentation using intelligent bioinspired clustering methods and k-means clustering presents in the next section, followed by materials and methods section that includes the proposed Whale Color Image based Segmentation Method (WhCIbS), then, section results and discusions that contain experiments that are compared proposed inspired Whale method with three bio-inspired clustering methods and K-means clustering methods. Finally, conclusions is explained in the last section. 


\section{LITERATURE REVIEW}

Image segmentation is defined as "a processing technique used to classify or cluster an image into several disjoint parts by grouping the pixels to form a region of homogeneity based on the pixel characteristics like gray level, color, texture, intensity and other features" (Khan \& Ravi, 2013).

The method for segmentation techniques in image are divided into two parts: Layer Based (LB) and Block Based (BB) (Zaitoun \& Aqel, 2015). The Layer Based method is not discussed in this paper, for more information about it refer to Juliet et al. (2014). The BB segmentation method analyses and understands the image depending on the diverse characteristics that appear in it. The BB segmentation method is classified into two parts: Edge Based and Region Based, where, there are very interesting research papers done in these two areas.

In Edge Based method, the image is transformed to edge image by changing tones in the image. On the other side, Rigon Based classified the image into sub regions (clusters) (Zaitoun \& Aqel, 2015). There are five categories of Rigon Based segmentation method; split and merge (Ning et al., 2010), normalized cuts (Shi \& Malik, 2000), region growing (Tang, 2010), threshold (Mishra \& Panda, 2018; Sarkar et al., 2011), and finally clustering (Alagarsamy et al., 2019; Zhang et al., 2011). This study is focused on the region based color image segmentation specified in clustering method.

Clustering method groups an image into k clusters of similar objects, homogeneous objects in one group while the heterogeneous objects are isolated. In literature, there are various works on medical image segmentation, satellite image segmentation, and color image segmentation that use traditional clustering methods combined with meta-heuristic algorithms.

In medical image segmentation, Dhanachandra, Manglen and Chanu (2015) proposed a method based on subtractive and k-means clustering algorithm, where, subtractive clustering algorithm found the initial centers from the data then k-means clustering algorithm works depending on these centers. After that, any unwanted regions are removed from segmentation image using medial filter. The result demonstrates that their proposed better than classical k-means algorithm in terms of PSNR and RMSE (Dhanachandra et al., 2015).

In satellite image segmentation, Kapoor et al. (2017) suggested satellite image segmentation method using the Grey wolf optimizer algorithm as an automatic clustering algorithm. The result of their proposed method is efficient in terms of Davies-Bouldinindex and inter-intra cluster distance.

In color image segmentation, Ye et al. (2003) proposed image segmentation method based on density based clustering. Nandy et al. (2015) proposed color image segmentation method optimized by Cuckoo Search algorithm CS. The researchers used CS algorithm to 
cluster the image after finding optimal centers (Nandy et al., 2015). Mathur and Purohit (2014) developed color image segmentation methods using k-means clustering algorithm, where, it classified the image into different clusters depending on Euclidean distance metric.

In the current algorithms, there has been a tendency to combining the inspired metaheuristic algorithms with clustering methods for improving the clustering performance. Although, some inspired meta-heuristic algorithms such as Particle Swarm Optimization has some disadvantages (i.e. low convergence rates and generating low quality solutions). This has happened because of the working mechanism of Particle Swarm Optimization keeping a single best solution through search space that can perform to local optima (Ghany et al., 2020).

Whale Optimization Algorithm is a well-known optimization algorithm. It has advantages such as minimum number of parameters, lack of local optima and acquiring a global optimal solution. These advantages lead WOA to become appropriate algorithm for finding the solutions of constrained or unconstrained optimization problems (Nasiri \& Khiyabani, 2018). Mirjalili and Lewi (2016) had examined WOA algorithm against other inspired metaheuristic algorithms like: PSO, Differential Evolutional, and other using twenty nine mathematical benchmark optimization problems. The results gave evidence that WOA a competitive with others (Mirjalili \& Lewis, 2016; Nasiri \& Khiyabani, 2018). Further, Jadhav and Gomathi (2018) proposed a WOA algorithm for data clustering based on three constraints termed inter-cluster distance, intra-cluster and the cluster density. Their proposed outperformed PSO, modified PSO, grey wolf optimization (Jadhav \& Gomathi, 2018). Also, Ghany et al. (2020) introduced combined WOA with Tabu search for data clustering. The results of their work can converge to global optima in less iteration, which it is better than PSO and other competitors (Ghany et al., 2020). In addition, Nasiri and Khiyabani (2018) proposed WOA algorithm for data collected from the UCI machine learning repository. Their proposing had shown success of WOA compared to PSO, ABC, GA, DE, and K-means (Nasiri \& Khiyabani, 2018).

Further, In literature, Whale Optimization Algorithm uses to determine the optimal multilevel threshold for image segmentation, wherethe researchers: El Aziz et al. (2017) used Whale Optimization Algorithm and moth flame Optimization with Otsu's objective function. While in another work, El Aziz et al. (2018) utilized Whale Optimization Algorithm with multi objective function for optimizing the threshold that used to split the image. Whale Optimization Algorithm is also used in the segmentation of medical image (i.e. liver image), where Mostafa et al. (2017) utilized manual segmented image dataset, then they converted them into binary images. The researchers used Whale Optimization Algorithm to cluster the intensity values in these images (Mostafa et al., 2017). Based on our knowledge, there is no work about utilizing Whale Optimization Algorithm for color image segmentation. 


\section{Whale Optimization Algorithm}

Whale Optimization Algorithm terms as WOA is a new bio-inspired optimization algorithm introduced by the researchers Mirjalili and Lewis in the year 2016 (Mirjalili \& Lewis, 2016). It is based on the natural hunting behavior of humpback whales. The humpback whale can dive around $12 \mathrm{~m}$ down a prey, then, creating bubbles net in a spiral shape and swim up towards the surface to hunt the prey. The humpback whale encircles the prey to preventing it from escaping. The pseudo code of WOA algorithm is shown in Figure 1 , where, the code is divided into three phases; encircle prey, search for prey, and spiral bubble net (El Aziz et al., 2017; Mirjalili \& Lewis, 2016; Mostafa et al., 2017).

In Encircle prey phase of WOA, humpback whale updates its position based on current best search agent (i.e. position of hunting prey). The other agents also update their position. This mechanism is represented mathematically in Equation 1 and 2 (Kaur \& Arora, 2018; Mirjalili \& Lewis, 2016).

$$
\begin{aligned}
& \vec{D}=\left|\vec{C} \cdot \overrightarrow{X^{*}}(t)-\vec{X}(t)\right| \\
& \vec{X}(t+1)=\overrightarrow{X^{*}}(t)-\vec{A} \cdot \vec{D}
\end{aligned}
$$

Where, $\vec{C}$ and $\vec{A}$ represent a coefficient vectors and they calculate using Equation 3 and 4 (Kaur \& Arora, 2018), t is the current iteration, $X^{*}$ indicates the best solution.

$$
\begin{aligned}
& \vec{A}=2 \vec{a} \cdot \vec{r}-\vec{a} \\
& \vec{C}=2 \cdot \vec{r}
\end{aligned}
$$

Where, $\vec{a}$ is a value minimizing from 2 to $0, \vec{r}$ is a random value between [0, 1].

In search for prey phase of WOA, humpback whale updates its position based on random position vector $\overrightarrow{X_{\text {rand }}}$ (i.e. random whale) that is chosen from the population. This phase is represented in Equation 5 and 6 (Kaur \& Arora, 2018).

$$
\begin{aligned}
& \vec{D}=\left|\vec{C} \cdot \overrightarrow{X_{\text {rand }}}-\vec{X}(t)\right| \\
& \vec{X}(t+1)=\overrightarrow{X_{\text {rand }}}-\vec{A} \cdot \vec{D}
\end{aligned}
$$

In the spiral bubble net phase of WOA, humpback whale mimics helix shaped movement when it changes its position towards the prey. The Equations 7 and 8 (Kaur \& Arora, 2018) represent this action.

$$
\begin{aligned}
& \overrightarrow{D^{\prime}}=\left|\overrightarrow{X^{*}}(t)-\vec{X}(t)\right| \\
& \vec{X}(t+1)=\overrightarrow{D^{\prime}} \cdot e^{b l} \cdot \cos (2 \pi l)+\overrightarrow{X^{*}}(t)
\end{aligned}
$$

Where, $\mathrm{b}$ is a constant value that represents the logarithmic spiral shape, 1 represents a random value between $[-1,1]$. The $\mathrm{p}$ value in the code represents a random value between $[0,1]$. 


\section{Initialization}

- Randomly initializing the whale population Xi, where $i=1,2, \ldots, n$

- Evaluating each search agent and calculate the fitness of them.

- Find best agent fitness $X^{*}$.

\section{Process}

- While iteration < Maximum iteration

- For each search agent

- Updating a, A, C, I, and P by using Equations 3 and 4

- If $(p<0.5)$

- If $(\operatorname{abs}(A)<1)$

\section{Encircle Prey}

- $\quad$ Updating current agent position using Equations 1 and 2

- Else if $(\operatorname{abs}(A)>=1)$

\section{Search for Prey}

- Selecting random agent $X_{\text {random }}$.

- Updating current agent position using Equations 5 and 6

- $\quad$ End if

\section{Bubble net}

- $\quad$ Else if $(p>=0.5)$

- Updating current agent position using Equation 7 and 8

- End if

- End For

- calculating the fitness of each search agent.

- Updating best agent fitness $X^{*}$.

- Iteration=iteration+1

- End while

- Return best solution $X^{*}$.

Figure 1. Pseudo code of Original Whale Optimization Algorithm (Kaur \& Arora, 2018) 


\section{MATERIALS AND METHODS}

The flowchart of proposed Whale Color Image based Segmentation method can be seen in Figure 2. In proposed WhCIbS method, some changes had conducted to the original WOA to make it suitable for working on clustering. The goal of proposed WhCIbS is to segment the color image into clusters using the whale optimization algorithm in clustering. The input to this method is color image in RGB color space and the output is the optimal centers that return optimal clusters.

The input image to the Whale clustering algorithm is converted into L*a*b color space. In the initialization of the Whale clustering algorithm, the number of $\mathrm{K}$ clusters is pre-defined, and the number of Whale population $\mathrm{n}$ is determined. Each whale holds a solution X, where it represents a set of centroids that generated randomly. Then the clusters are constructed based on these centers by assigning a data value into clusters. The creating clusters are based on calculating the mean values of the attributes of the objects within their given clusters.

For each whale, the fitness value is computed based on Equation 9 (Tang et al., 2012). After that, the best whale is selected that has minimum fitness value and its corresponding position $\mathrm{X}^{*}$. Where, the data point belong to i clusters has minimum distance from i center.

$$
F(C)=\sum_{i=1}^{k} \sum_{j=1}^{S} w_{j, i} \sum_{o=1}^{k * D}\left(X_{j, o}-C_{i, o}\right)^{2}
$$

Where, $\mathrm{C}$ is array containing cluster centers, $\mathrm{k}$ is the number of clusters, $\mathrm{s}$ is all solutions of $\mathrm{X}, \mathrm{w}$ is set to 1 if $\mathrm{x}$ belongs to the cluster, otherwise $\mathrm{w}$ is set to $0, \mathrm{D}$ is the search space dimension.

The Whale attacks their prey in either encircling or bubble-net strategies. The attacking is based on $\mathrm{P}$ value that represents a random number using for determining the probability of selecting one of two strategies. If the $\mathrm{P}$ is less than 0.5 the encircling step starts otherwise the bubble-net step starts.

In encircling step, the position of the whale is updated based on parameter A that can be calculated using Equation 3. If the value of A less than 1 the Whale updates the position based on best position $\mathrm{X}^{*}$. Otherwise, the Whale updates their position based on choosing a random search agent. This process is depending on Equation 5 and 6 .

If the value of $\mathrm{P}$ is greater or equal 0.5 then the bubble-net strategy using Equation 7 and 8 is conducted to update the positions. The process of calculating the fitness value of whales and updating the best agent fitness continues until reaching the maximum number of iterations that is determined initially in the starting of the algorithm.

The output of the Whale clustering algorithm is optimal clusters to segment the color image. Then, the measuring segmented images using performance metrics are conducted. The performance of metrics equations is shown in the following results and discussion section. 


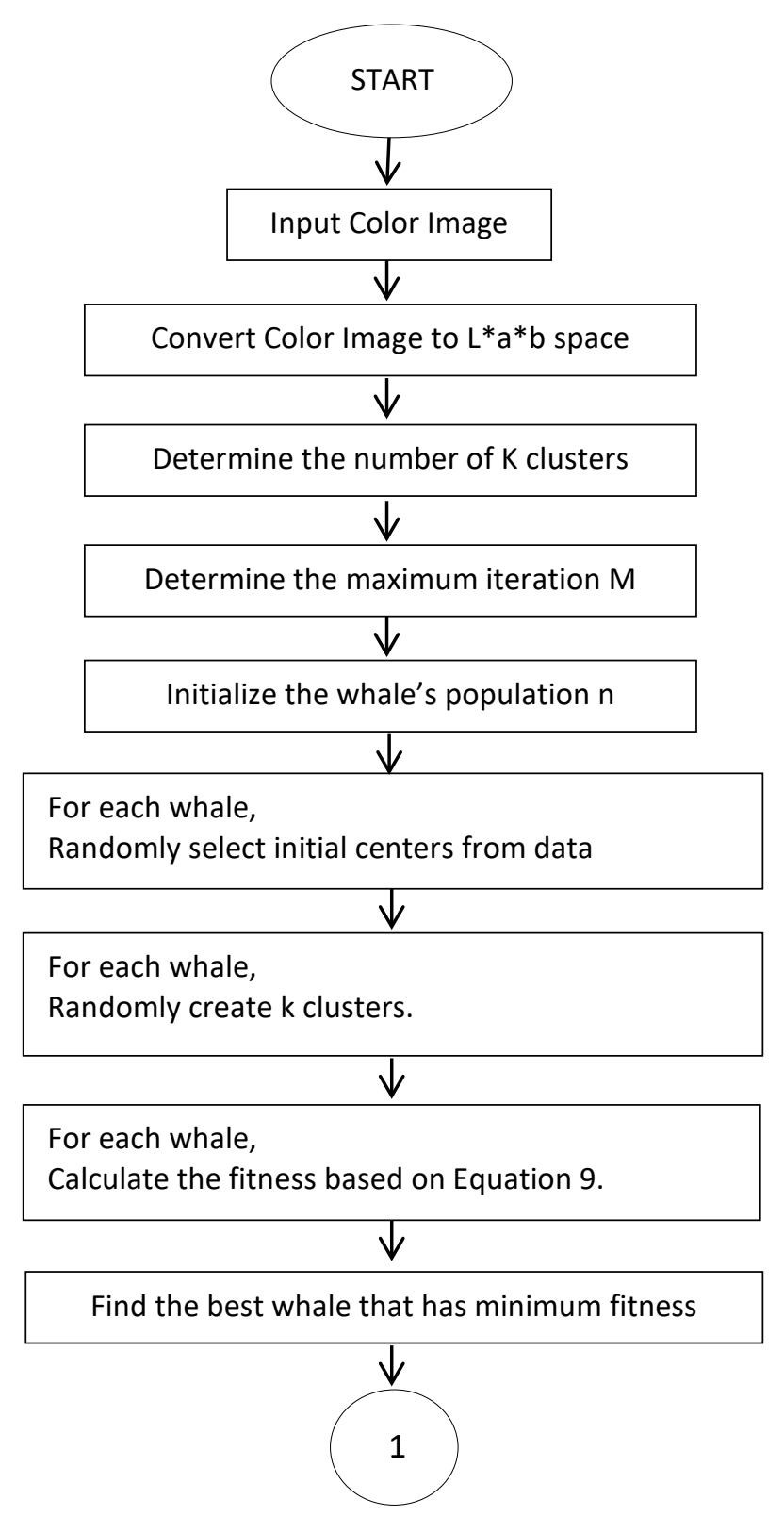

Figure 2. The flowchart of proposed Whale Color Image based Segmentation method 


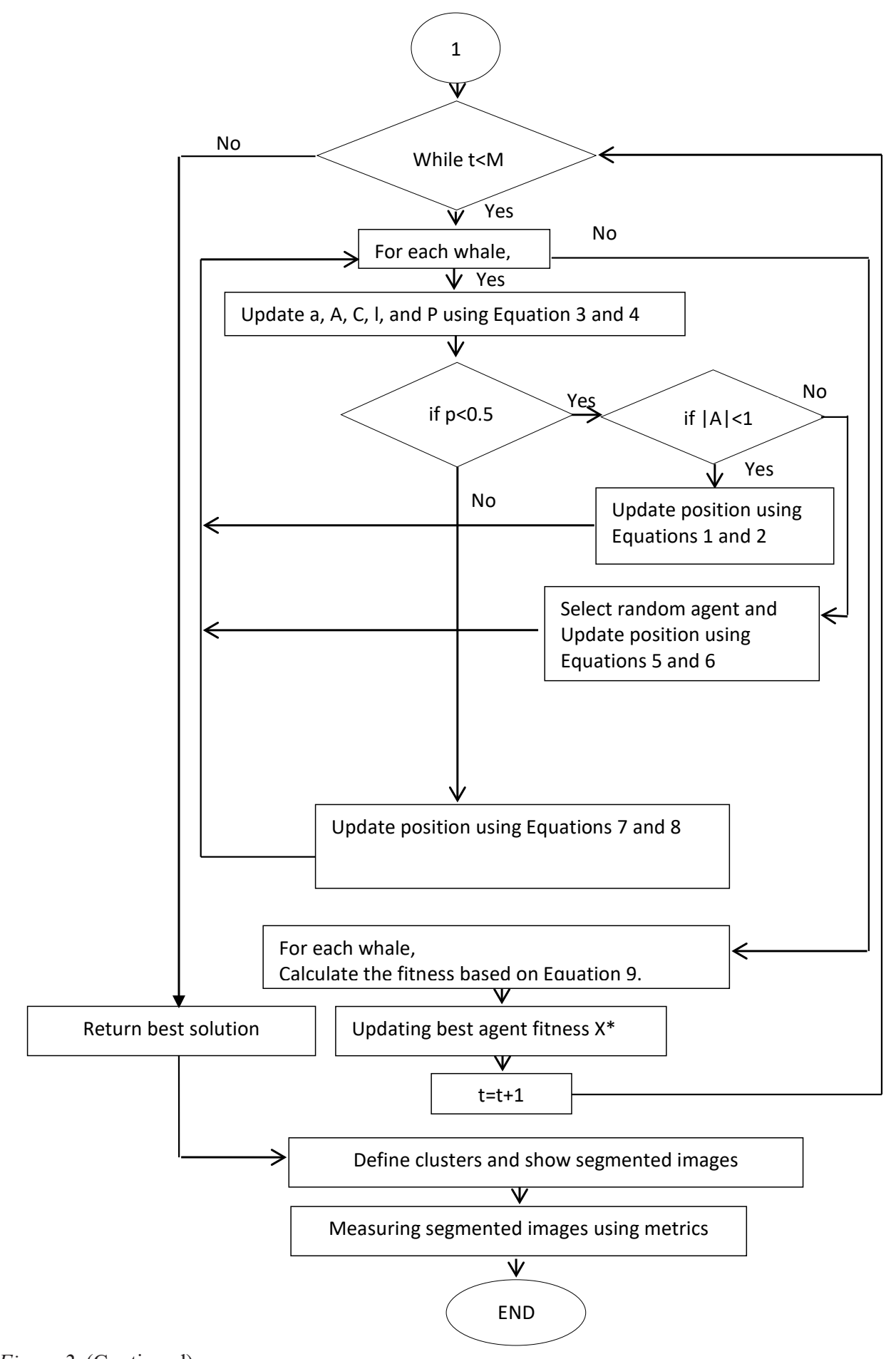

Figure 2. (Continued) 


\section{RESULTS AND DISCUSIONS}

In this section, the environment of the experiments for the proposed Whale Color Image based Segmentation method is introduced. Firstly, the description of benchmark images is illustrated. Then, the parameters setup for proposed and comparative methods is briefly explained. Then the performance metrics for measuring the segmentation quality are described, followed by the experimental results.

\section{Benchmark Images}

In this paper, four test color images are utilized to test the performance of the proposed WhCIbS method. These images were collected from Berkeley Segmentation Dataset: Training Image ( https://www2.eecs.berkeley.edu/Research/Projects/CS/vision/bsds/ BSDS300/html/dataset/images/color/train-076-100.html). The original test color images were named in the experiment; Deer, Dog, Lake, and Bear and they are shown in Figure 3.

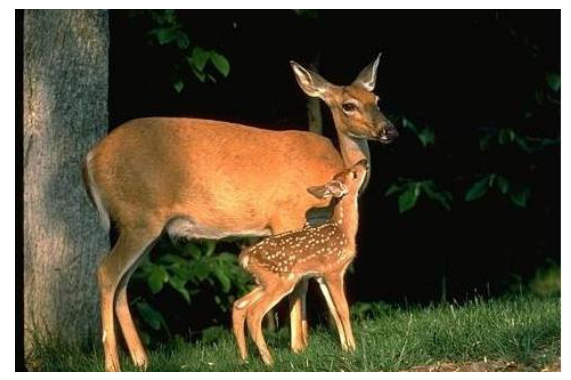

(a)

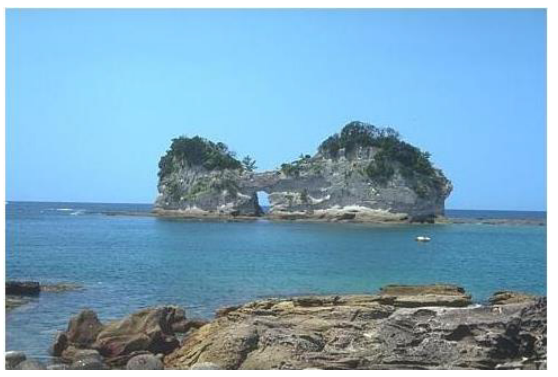

(c)

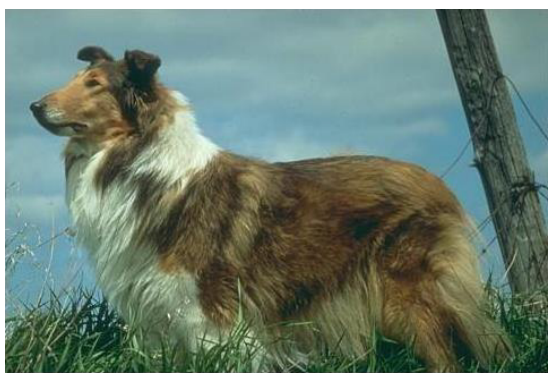

(b)

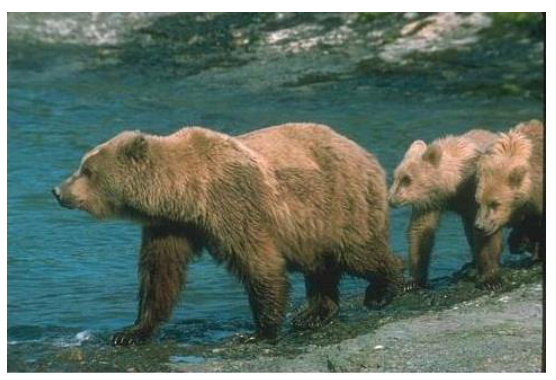

(d)

Figure 3. Original test image (a) Deer (b) Dog (c) Lake (d) Bear.

\section{Parameters Setup}

The proposed Whale Color Image based Segmentation method WhCIbS was preformed in MATLAB version 2018 in 64-bit windows 8 operating system with Intel Core i3 Duo $1.80 \mathrm{GHz}$ processor. The results of proposed WhCIbS were compared with four methods; Wolf Color Image based Segmentation (WCIbS), Cuckoo Color Image based Segmentation 
(CCIbS), BatColor Image based Segmentation (BCIbS) and K-means Color Image based Segmentation (KmCIbS) (Mathur \& Purohit, 2014). For a fair comparison all methods had similar number of iterations, similar size of population and all methods testing on same test images. The parameters setup of the experiment for intelligent inspired optimization algorithms; Wolf, Cuckoo Search, Bat, and Whale algorithms are shown in Table 1.

Table 1

Parameters of the Wolf, Cuckoo, Bat, and Whale algorithms

\begin{tabular}{|c|c|c|c|c|}
\hline Parameters & $\begin{array}{l}\text { Wolf } \\
\text { algorithm }\end{array}$ & $\begin{array}{l}\text { Cuckoo } \\
\text { algorithm }\end{array}$ & Bat algorithm & $\begin{array}{l}\text { Whale } \\
\text { algorithm }\end{array}$ \\
\hline Number of population & 20 & 20 & 20 & 20 \\
\hline Visual distance & 1 & - & - & - \\
\hline Escape distance & 5 & - & - & - \\
\hline Escape probability & 0.25 & - & - & - \\
\hline Discovery rate & - & 0.25 & - & - \\
\hline$\beta$ & - & 1.5 & - & - \\
\hline A: loudness & - & - & 0.5 & - \\
\hline R: pulse rate & - & - & 0.5 & - \\
\hline $\begin{array}{l}\text { Qmin: minimum } \\
\text { frequency }\end{array}$ & - & - & 0 & - \\
\hline $\begin{array}{l}\text { Qmax: maximum } \\
\text { frequency }\end{array}$ & - & - & 0.2 & - \\
\hline $\mathrm{a}$ & - & - & - & {$[0-2]$} \\
\hline $\mathrm{b}$ & - & - & - & 1 \\
\hline 1 & - & - & - & {$[-1,1]$} \\
\hline
\end{tabular}

\section{Performance Metrics}

Two performance metrics were used for evaluating the quality of the segmented images by the proposed WhCIbS and the comparative methods as follows; the Peak Signal to Noise Ratio (PSNR) which it was utilized for measuring the difference between segmented and reference images. The higher value of PSNR is the better performance of segmentation (Dhanachandra et al., 2015; El Aziz et al., 2017). Equation 10 shows the PSNR metric.

$$
P S N R=10 \log _{10} \frac{\max [g(x, y)]^{2}}{R M S E}
$$

The second metric was the Root Mean Squared Error (RMSE). The lower value of RMSE means better performance of segmentation (Dhanachandra et al., 2015; El Aziz et al., 2017). Equation 11 shows the RMSE metric. 


$$
R M S E=\sqrt{\frac{\sum_{0}^{m x-1} \sum_{0}^{m y-1}[g(x, y)]^{2}}{m x m y \sum_{0}^{m x-1} \sum_{0}^{m y-1}[g(x, y)-s(x, y)]^{2}}}
$$

Where, $g(x, y)$ represents the test image and $s(x, y)$ represents the segmented image of size (mxXmy).

\section{Experimental Results}

The results of the proposed Whale Color Image based Segmentation method WhCIbS and the comparative methods are clarified in Table 2.

As can see in Table 2 the PSNR of the proposed WhCIbS is highlighted in Table 2 (36.030, 38.801, 38.762, 38.868) for all test images: deer, dog, lake, and bear respectively, which is is a better result in most cases than the comparative methods WCIbS, CCIbS, $\mathrm{BCIbS}$, and $\mathrm{KmCIbS}$. Higher value of PSNR means better performance of segmentation (Dhanachandra et al., 2015; El Aziz et al., 2017). Figure 4 to 7 present the comparative results of PSNR for five methods WCIbS, CCIbS, BCIbS, WhCIbS and KmCIbS for four test images.

Table 2

Results of PSNR and RMSE for five methods: WCIbS, CCIbS,BCIbS, WhCIbS and KmCIbS.

\begin{tabular}{llll}
\hline Image name & Methods & PSNR & RMSE \\
\hline Deer & WCIbS & 36.023 & 10.572 \\
& CCIbS & 36.012 & 10.576 \\
BCIbS & 36.025 & 10.571 \\
WhCIbS & 36.030 & $\mathbf{1 0 . 5 6 9}$ \\
Dog & KmCIbS & 36.025 & 10.571 \\
& WCIbS & 38.740 & 12.999 \\
& CCIbS & 38.713 & 13.012 \\
& BCIbS & 38.741 & 12.999 \\
Lake & WhCIbS & $\mathbf{3 8 . 8 0 1}$ & $\mathbf{1 2 . 9 6 9}$ \\
& KmCIbS & 38.741 & 12.999 \\
& WCIbS & 38.745 & 13.036 \\
& CCIbS & 38.739 & 13.038 \\
& BCIbS & 38.745 & 13.035 \\
WhCIbS & $\mathbf{3 8 . 7 6 2}$ & $\mathbf{1 3 . 0 2 7}$ \\
\hline
\end{tabular}


Table 2 (continued)

\begin{tabular}{llll}
\hline Image name & Methods & PSNR & RMSE \\
\hline Bear & BCIbS & 38.822 & 12.993 \\
& WhCIbS & $\mathbf{3 8 . 8 6 8}$ & $\mathbf{1 2 . 9 6 9}$ \\
& KmCIbS & 38.819 & 12.995 \\
\hline
\end{tabular}

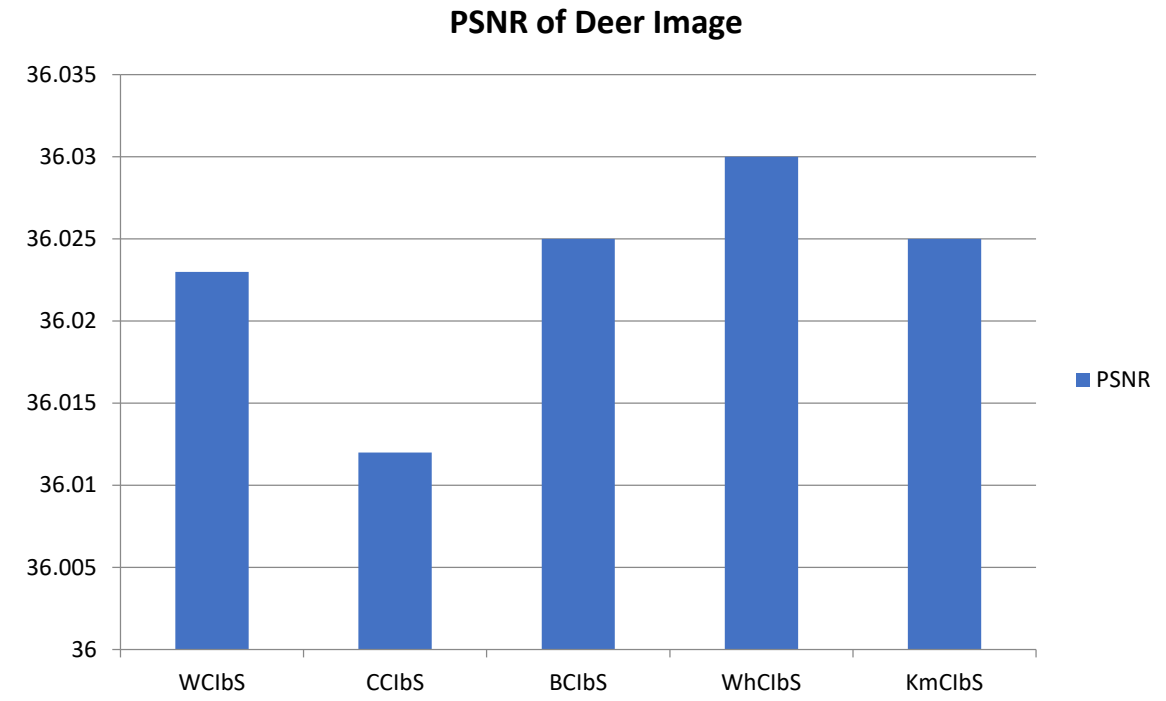

Figure 4. PSNR of five methods WCIbS, CCIbS,BCIbS, WhCIbS and KmCIbS for Deer image.

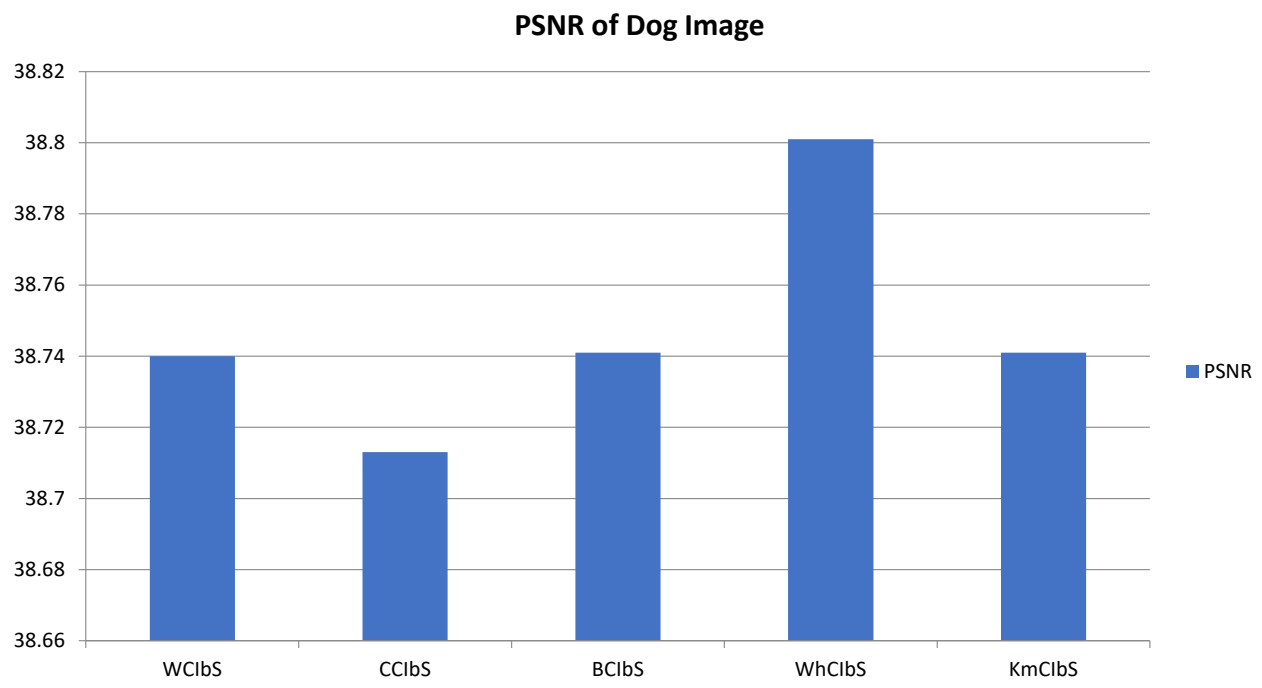

Figure 5. PSNR of five methods WCIbS, CCIbS,BCIbS, WhCIbS and KmCIbS for Dog image. 


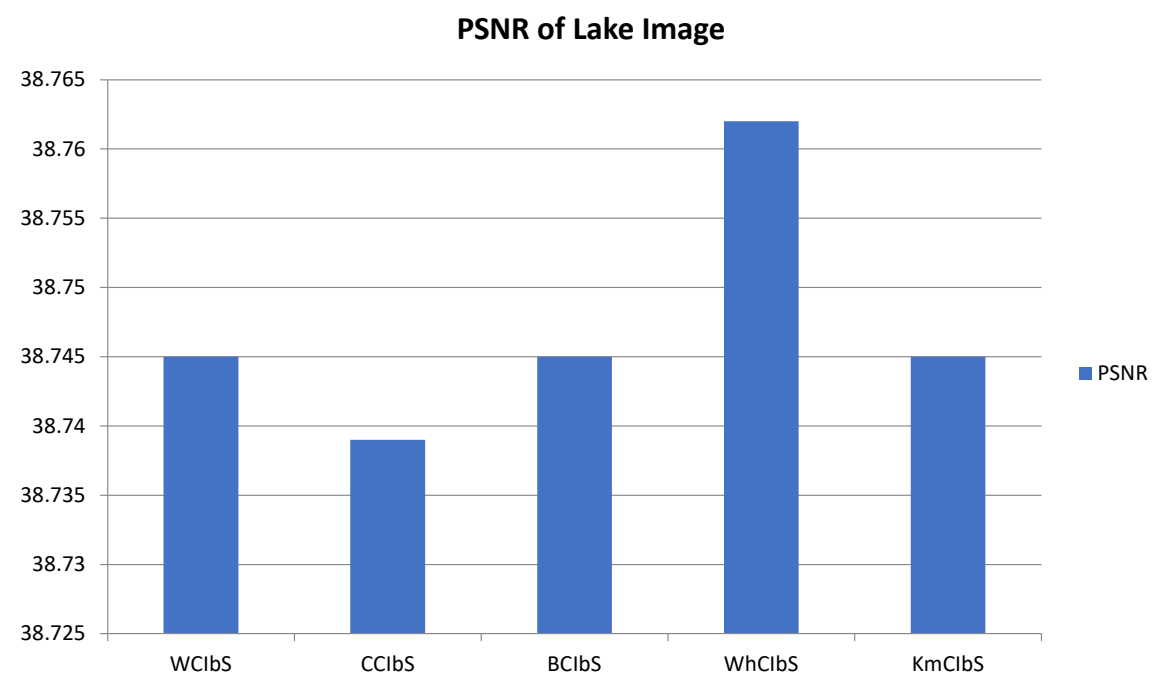

Figure 6. PSNR of five methods WCIbS, CCIbS,BCIbS, WhCIbS and KmCIbS for Lake image.

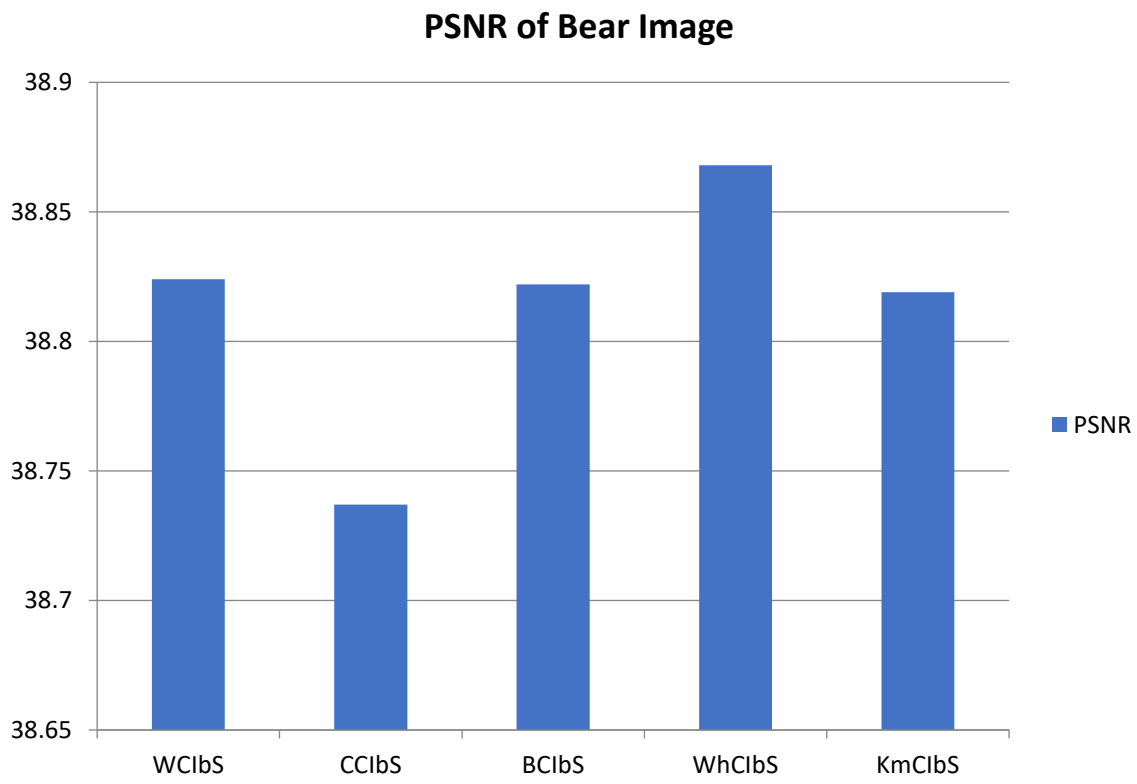

Figure 7. PSNR of five methods WCIbS, CCIbS,BCIbS, WhCIbS and KmCIbS for Bear image.

The RMSE for proposed WhCIbS and all comparative methods can be observed in Table 2. The highlighted values in Table 2, 10.569, 12.969, 13.027, 12.969 for proposed WhCIbS are minimum values of RMSE compared than other methods; WCIbS, CCIbS, $\mathrm{BCIbS}$ and $\mathrm{KmCIbS}$. Lower value of RMSE means better quality of segmentation 
(Dhanachandra et al., 2015; El Aziz et al., 2017). Figure 8 to 11 exhibit RMSE of five methods WCIbS, CCIbS, BCIbS, WhCIbS and KmCIbS for four test images.

\section{RMSE of Deer Image}

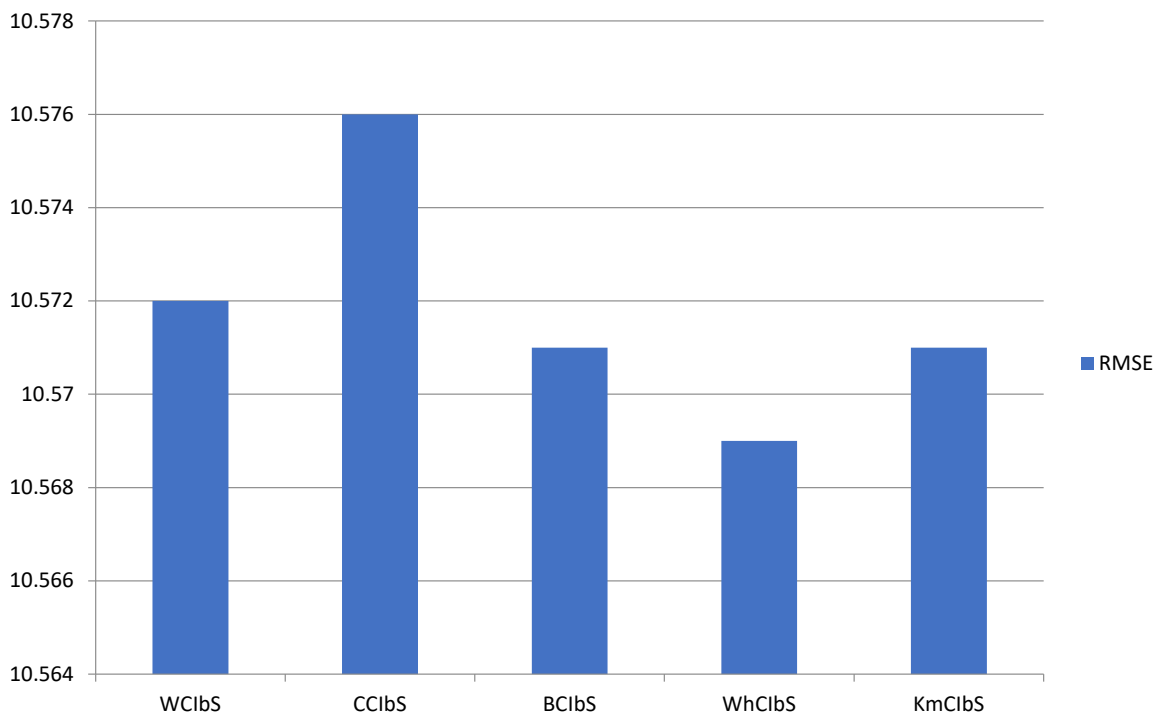

Figure 8. RMSE of five methods WCIbS, CCIbS, BCIbS, WhCIbS and KmCIbS for Deer image.

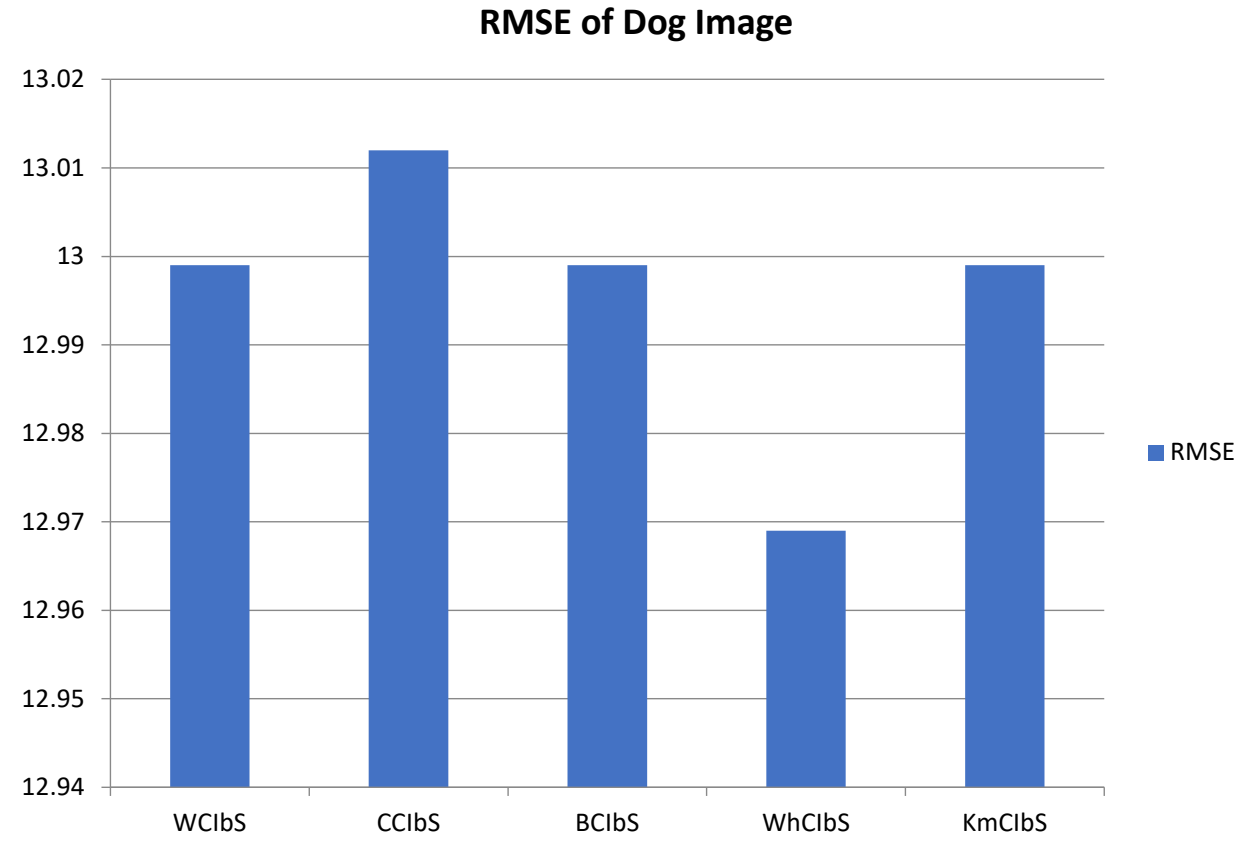

Figure 9. RMSE of five methods WCIbS, CCIbS, BCIbS, WhCIbS and KmCIbS for Dog image. 
RMSE of Lake Image

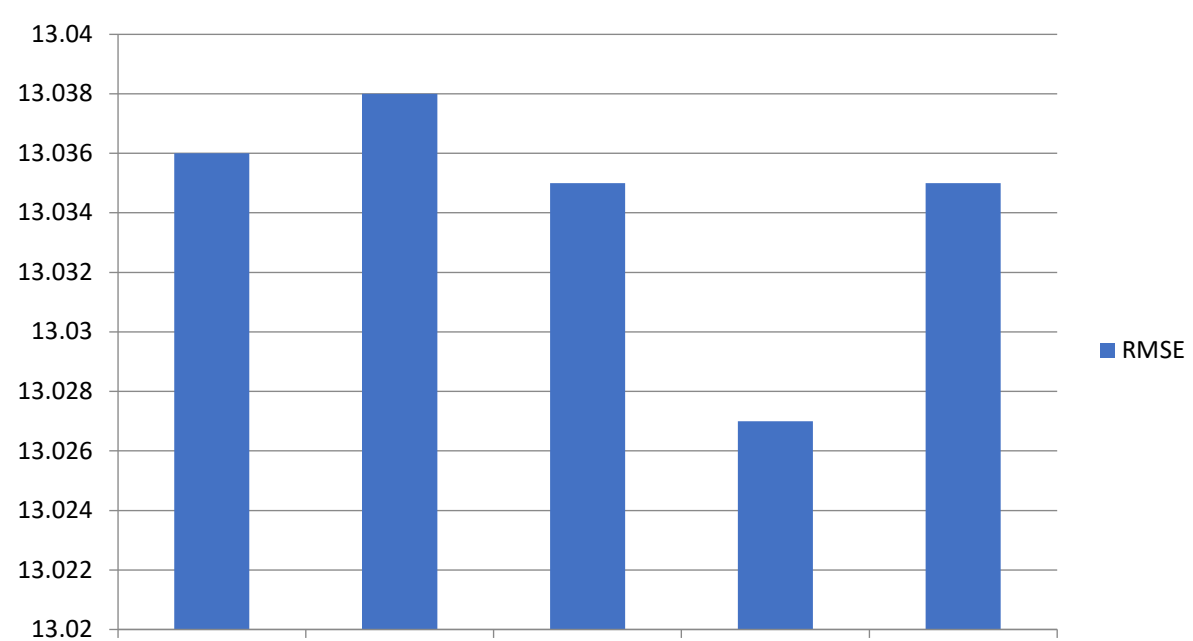

Figure 10. RMSE of five methods WCIbS, CCIbS, BCIbS, WhCIbS and KmCIbS for Lake image.

RMSE of Bear Image

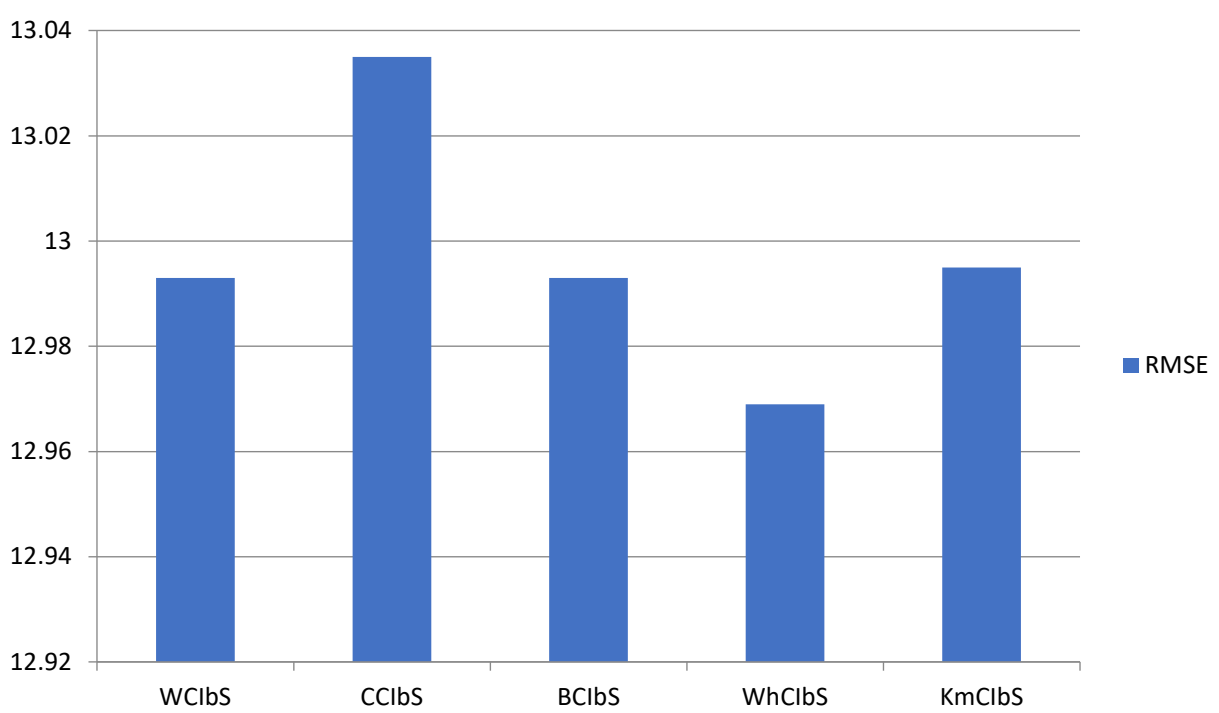

Figure 11. RMSE of five methods WCIbS, CCIbS, BCIbS, WhCIbS and KmCIbS for Bear image.

Figure 12 to 15 show color image segmentation for all methods. They indicate that WhCIbS is performed better whenever $\mathrm{k}=3$. 


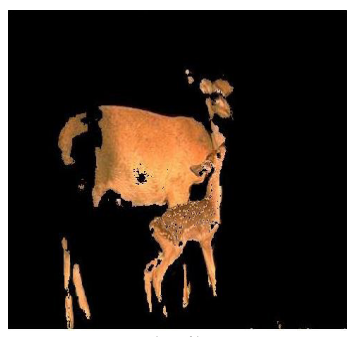

(a.1)

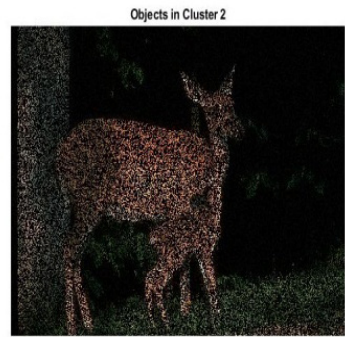

(a.4)
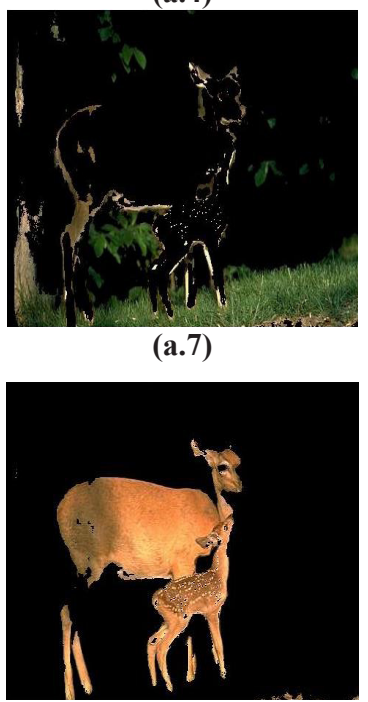

(a.10)

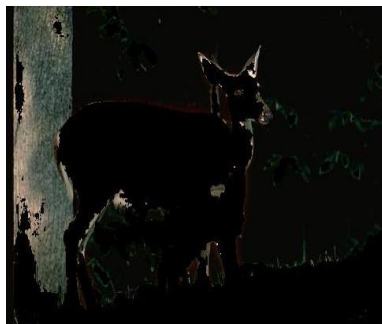

(a.13)

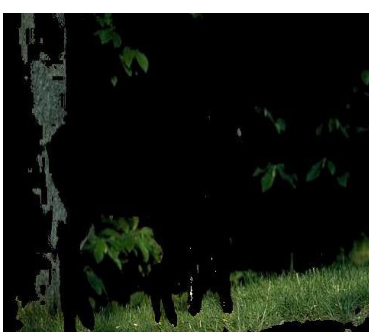

(a.2)

Objects in Cluster 2

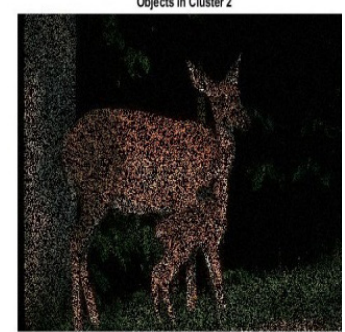

(a.5)

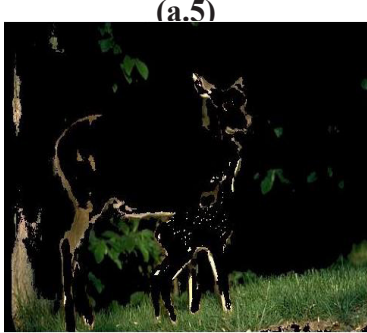

(a.8)

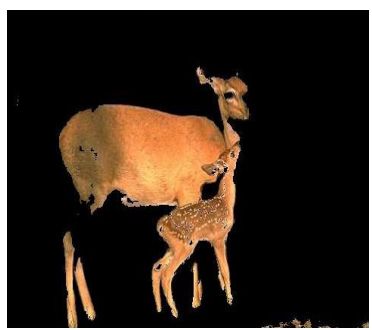

(a.11)

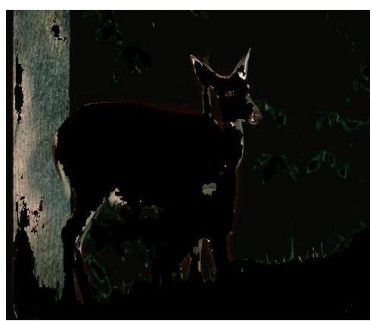

(a.14)

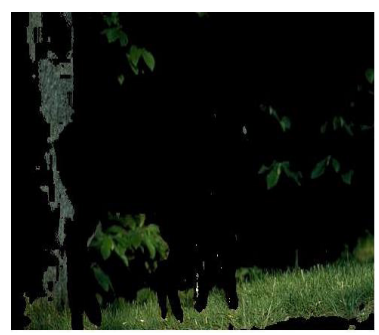

(a.3)

Objects in Cluster 3

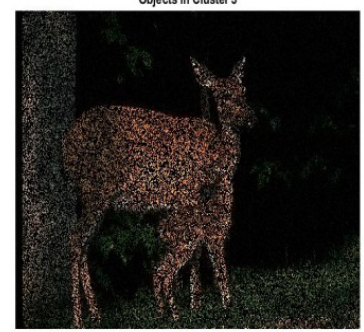

(a.6)

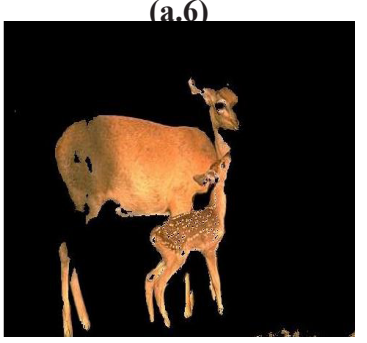

(a.9)

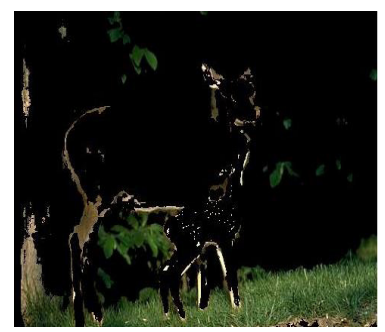

(a.12)

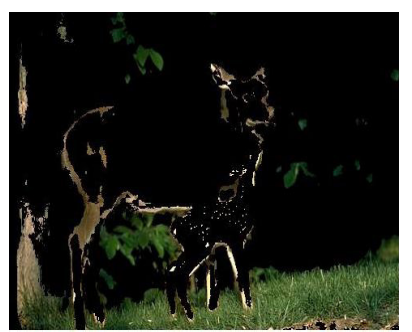

(a.15)

Figure 12. Color image segmentation[(a.1) (a.2) (a.3)] obtain by WCIbS method, [(a.4) (a.5) (a.6)] obtained by CCIbS method,[(a.7) (a.8) (a.9)] obtained by BCIbS method,[(a.10) (a.11) (a.12)] obtained by WhCIbS method, and [(a.13) (a.14) (a.15)] obtained by KmCIbS method. 
Athraa Jasim Mohammed and Khalil Ibrahim Ghathwan

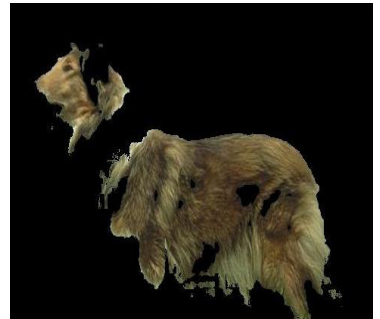

(b.1)

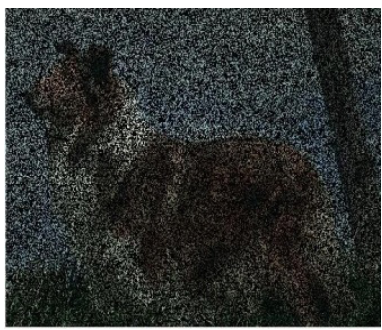

(b.4)

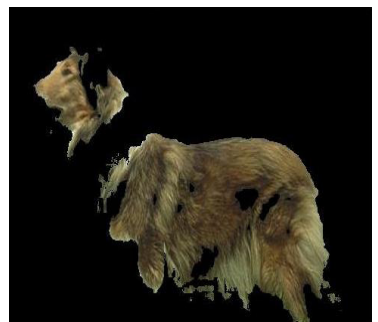

(b.7)

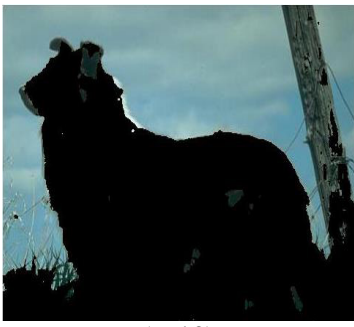

(b.10)

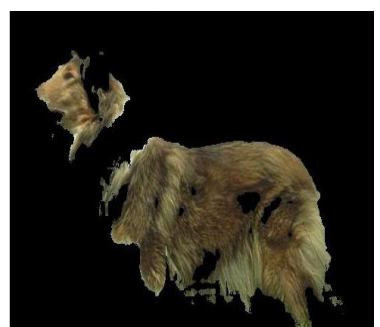

(b.13)

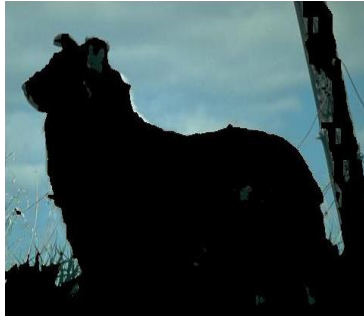

(b.2)

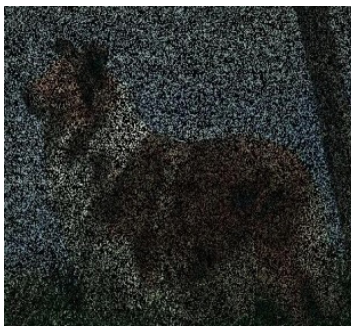

(b.5)

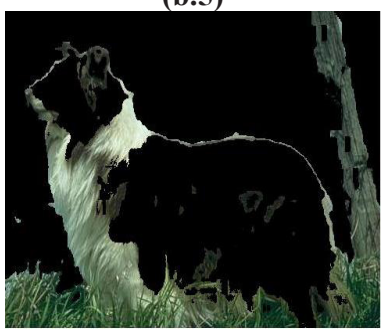

(b.8)

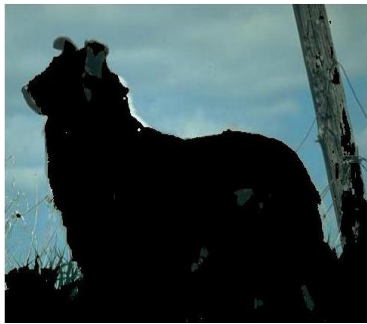

(b.11)

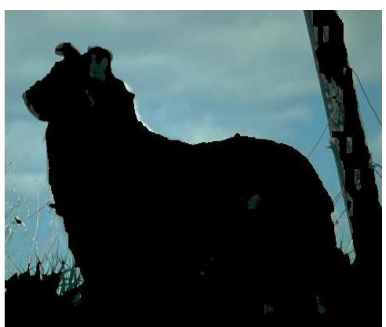

(b.14)

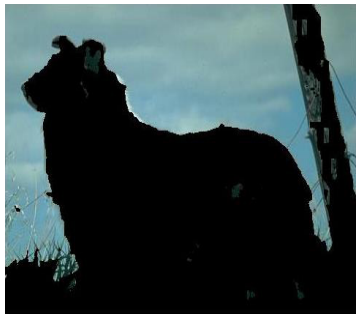

(b.3)

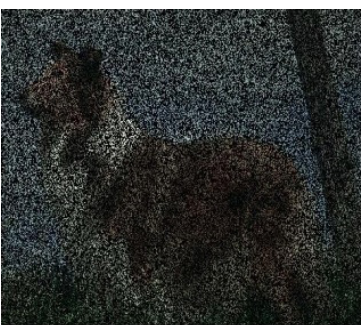

(b.6)

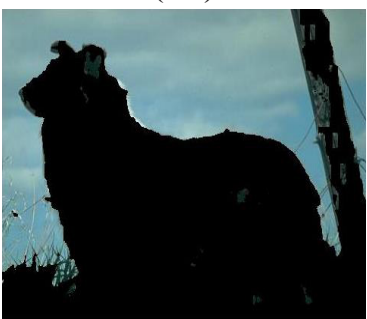

(b.9)

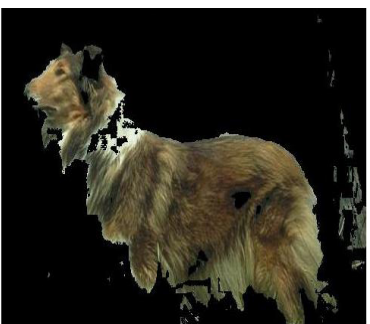

(b.12)

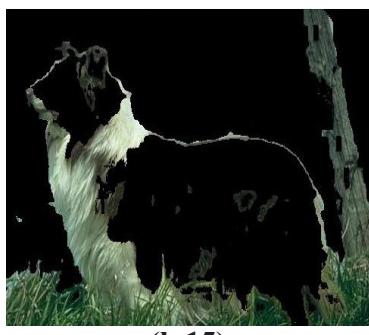

(b.15)

Figure 13. Color image segmentation[(b.1) (b.2) (b.3)] obtain by WCIbS method, [(b.4) (b.5) (b.6)] obtained by CCIbS method,[(b.7) (b.8) (b.9)] obtained by BCIbS method, [(b.10) (b.11) (b.12)] obtained by WhCIbS method, and [(b.13) (b.14) (b.15)] obtained by KmCIbS method. 


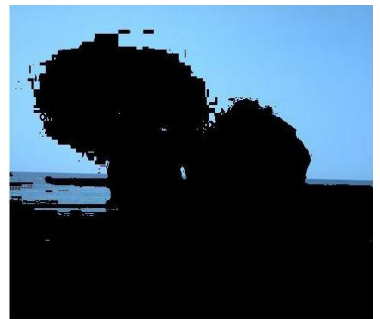

(c.1)

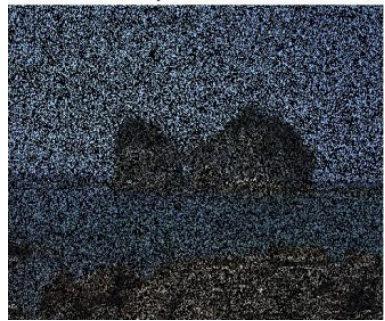

(c.4)

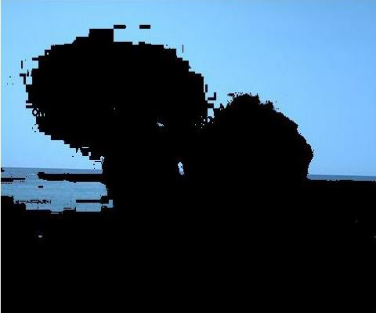

(c.7)

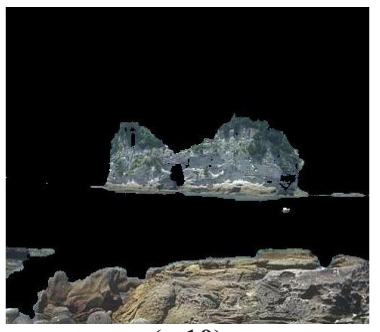

(c.10)

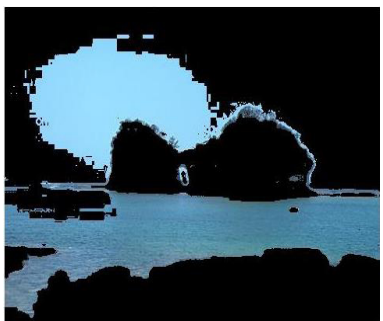

(c.13)

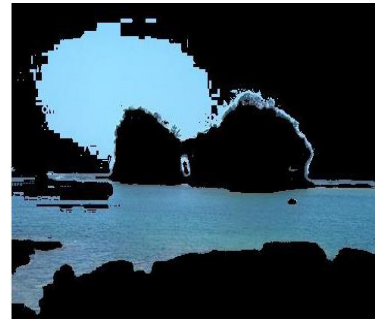

(c.2)

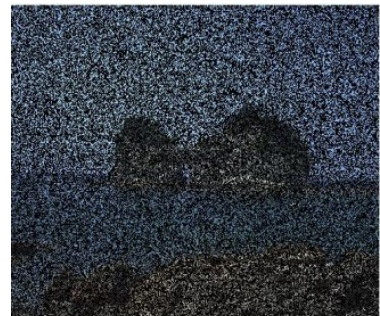

(c.5)

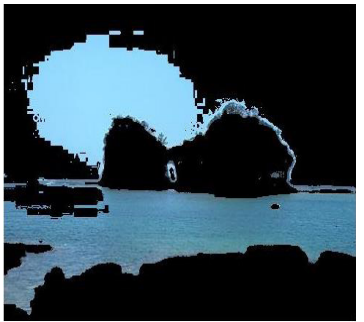

(c.8)

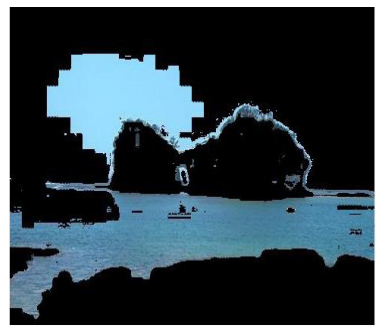

(c.11)

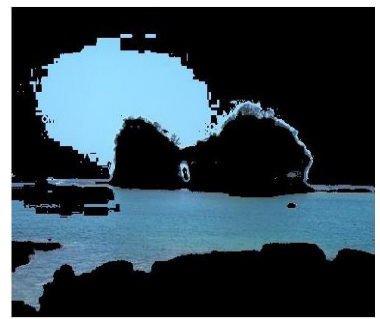

(c.14)

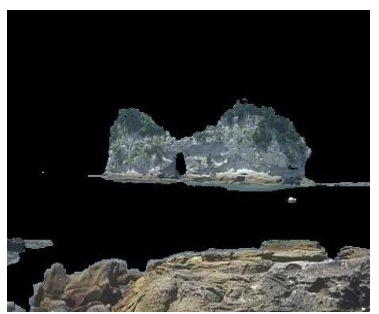

(c.3)

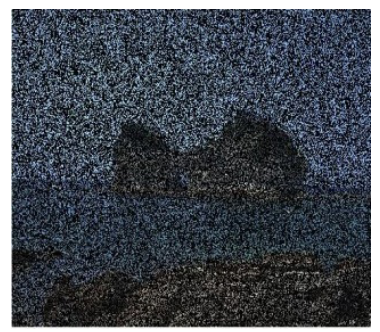

(c.6)

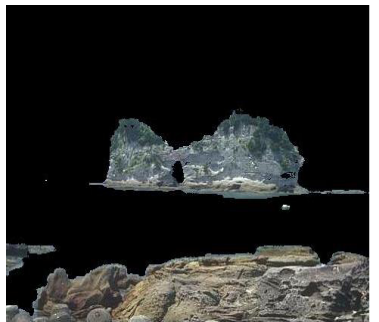

(c.9)

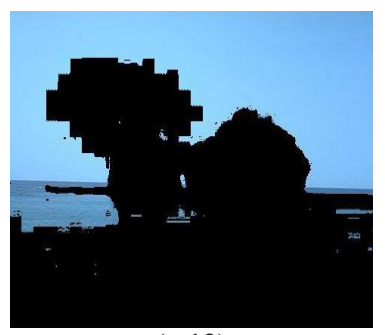

(c.12)

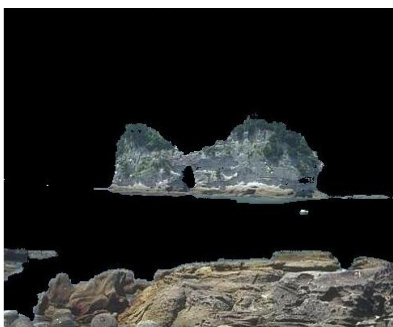

(c.15)

Figure 14. Color image segmentation[(c.1) (c.2) (c.3)] obtain by WCIbS method, [(c.4) (c.5) (c.6)] obtained by CCIbS method,[(c.7) (c.8) (c.9)] obtained by BCIbS method,[(c.10) (c.11) (c.12)] obtained by WhCIbS method, and [(c.13) (c.14) (c.15)] obtained by KmCIbS method. 
Athraa Jasim Mohammed and Khalil Ibrahim Ghathwan

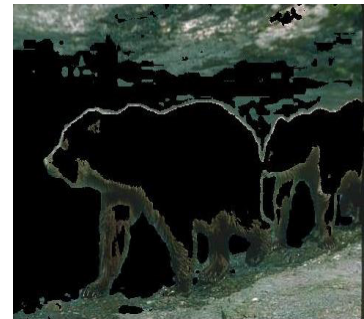

(d.1)

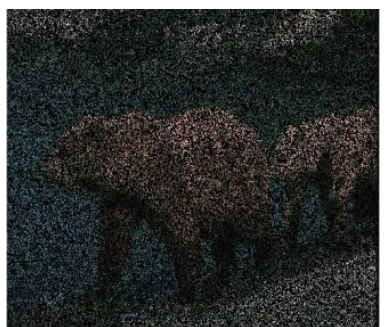

(d.4)

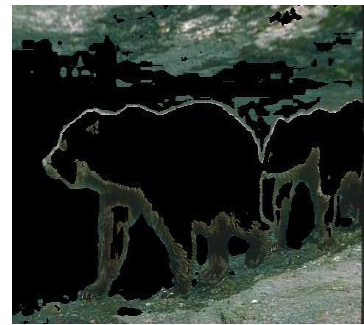

(d.7)

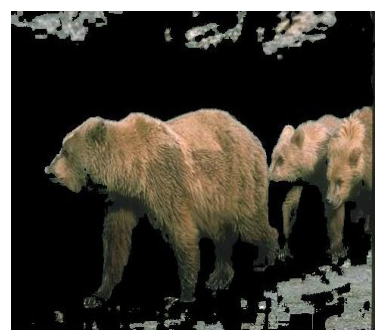

(d.10)

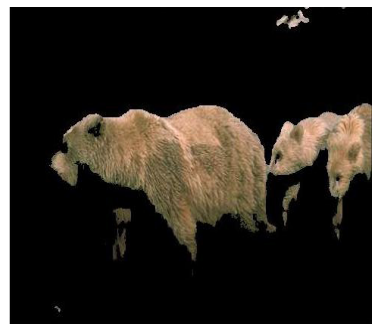

(d.13)

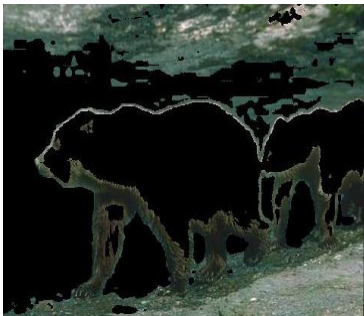

(d.2)

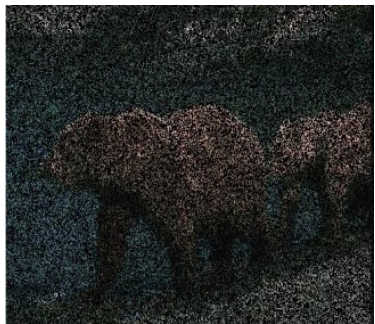

(d.5)

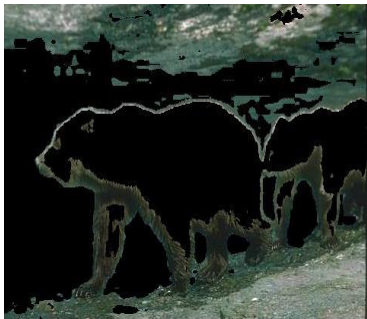

(d.8)

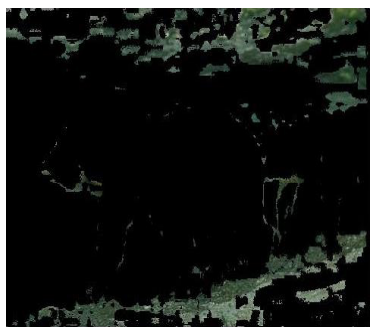

(d.11)

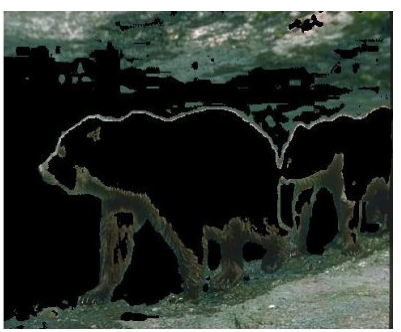

(d.14)

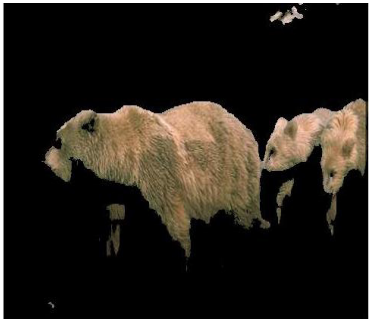

(d.3)

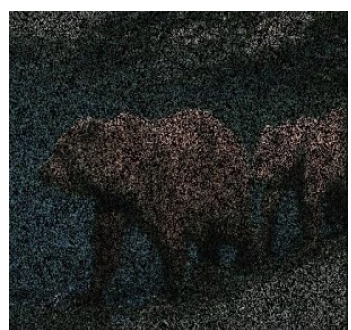

(d.6)

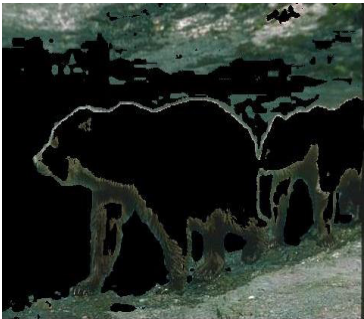

(d.9)

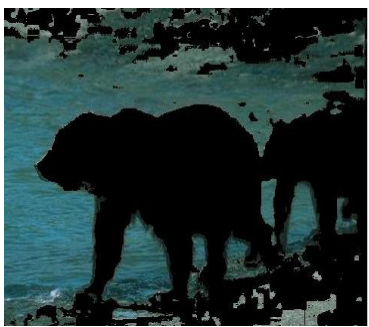

(d.12)

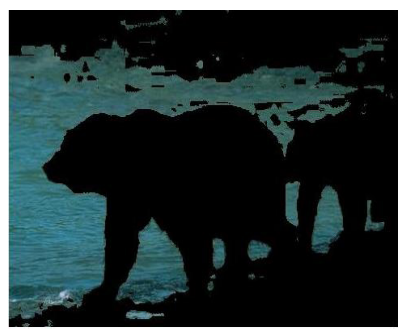

(d.15)

Figure 15. Color image segmentation[(d.1) (d.2) (d.3)] obtain by WCIbS method, [(d.4) (d.5) (d.6)] obtained by CCIbS method,[(d.7) (d.8) (d.9)] obtained by BCIbS method, [(d.10) (d.11) (d.12)] obtained by WhCIbS method, and [(d.13) (d.14) (d.15)] obtained by KmCIbS method. 


\section{CONCLUSIONS}

Color image segmentation using a clustering technique is still an open problem. The determining of the optimal clusters has been considered as an optimization problem. Hence, a new swarm algorithm called the Whale optimization algorithm has been proposed to find a sloution for this problem using Euclidean distance as objective function. The goal of this algorithm is to obtain the best clusters for input color image. The proposed Whale Color Image based Segmentation (WhCIbS) method has been compared with three swarm algorithms; wolf color image based segmentation, cuckoo color image based segmentation, and bat color image based segmentation. Further, it is compared with k-means color image based segmentation. The performance of the proposed method and the comparativemethods has been evaluated based on two quality metrics; PSNR and RMSE. Experiments have been used four benchmark images that collected from the Berkeley segmentation dataset. The results demonstrate that the proposed method achieved better performance than the comparative methods in terms of PSNR and RMSE. In the future, we will study the performance of the proposed method with multi objective functions in order to obtain better quality outcomes for image segmentation.

\section{ACKNOWLEDGEMENT}

This research received no specific grant from any funding agency in the public, commercial, or not-for-profit sectors.

\section{REFERENCES}

Alagarsamy, S., Kamatchi, K., Govindaraj, V., Zhang, Y. D., \& Thiyagarajan, A. (2019). Multi-channeled MR brain image segmentation: A new automated approach combining BAT and clustering technique for better identification of heterogeneous tumors. Biocybernetics and Biomedical Engineering, 39(4), 1005-1035.

Dhanachandra, N., Manglem, K., \& Chanu, Y. J. (2015). Image segmentation using K-means clustering algorithm and subtractive clustering algorithm. Procedia Computer Science, 54, 764-771.

El Aziz, M. A., Ewees, A. A., \& Hassanien, A. E. (2017). Whale optimization algorithm and moth-flame optimization for multilevel thresholding image segmentation. Expert Systems with Applications, 83, 242-256.

El Aziz, M. A., Ewees, A. A., Hassanien, A. E., Mudhsh, M., \& Xiong, S. (2018). Multi-objective whale optimization algorithm for multilevel thresholding segmentation. In Advances in soft computing and machine learning in image processing (pp. 23-39). Cham, Switzerland: Springer.

Ghany, K. K. A., AbdelAziz, A. M., Soliman, T. H. A., \& Sewisy, A. A. E. M. (2020, In Press). A hybrid modified step whale optimization algorithm with tabu search for data clustering. Journal of King Saud University - Computer and Information Sciences. DOI: https://doi.org/10.1016/j.jksuci.2020.01.015

Gonzalez, R. C., Woods, R. E., \& Eddins, S. L. (2003). Digital image processing using MATLAB. Upper Saddle River, USA: Prentice-Hall, Inc. 
Jadhav, A. N., \& Gomathi, N. (2018). WGC: Hybridization of exponential grey wolf optimizer with whale optimization for data clustering. Alexandria Engineering Journal, 57(3), 1569-1584.

Juliet, S. E., Sadasivam, V., \& Florinabel, D. J. (2014). Effective layer-based segmentation of compound images using morphology. Journal of Real-Time Image Processing, 9(2), 299-314.

Kapoor, S., Zeya, I., Singhal, C., \& Nanda, S. J. (2017). A grey wolf optimizer based automatic clustering algorithm for satellite image segmentation. Procedia Computer Science, 115, 415-422.

Kaur, G., \& Arora, S. (2018). Chaotic whale optimization algorithm. Journal of Computational Design and Engineering, 5(3), 275-284.

Khan, A. M., \& Ravi, S. (2013). Image segmentation methods: A comparative study. International Journal of Soft Computing and Engineering, 3(4), 84-92.

Mafarja, M. M., \& Mirjalili, S. (2017). Hybrid whale optimization algorithm with simulated annealing for feature selection. Neurocomputing, 260, 302-312.

Mathur, G., \& Purohit, H. (2014). Performance analysis of color image segmentation using k-means clustering algorithm in different color spaces. IOSR Journal of VLSI and Signal Processing, 4, 1-4.

Mirjalili, S., \& Lewis, A. (2016). The Whale Optimization Algorithm. Advances in Engineering Software, 95, 51-67.

Mishra, S., \& Panda, M. (2018). Bat algorithm for multilevel colour image segmentation using entropy-based thresholding. Arabian Journal for Science and Engineering, 43(12), 7285-7314.

Mostafa, A., Hassanien, A. E., Houseni, M., \& Hefny, H. (2017). Liver segmentation in MRI images based on whale optimization algorithm. Multimedia Tools and Applications, 76(23), 24931-24954.

Nandy, S., Yang, X., Sarkar, P. P., \& Das, A. (2015). Color image segmentation by cuckoo search. Intelligent Automation and Soft Computing, 21(4), 673-685.

Nasiri, J., \& Khiyabani, F. M. (2018). A whale optimization algorithm (WOA) approach for clustering. Cogent Mathematics and Statistics, 5(1), 1-13.

Ning, J., Zhang, L., Zhang, D., \& Wu, C. (2010). Interactive image segmentation by maximal similarity based region merging. Pattern Recognition, 43(2), 445-456.

Sarkar, S., Patra, G. R., \& Das, S. (2011). A differential evolution based approach for multilevel image segmentation using minimum cross entropy thresholding. In International Conference on Swarm, Evolutionary, and Memetic Computing (pp. 51-58). Heidelberg, Germany: Springer.

Shi, J., \& Malik, J. (2000). Normalized cuts and image segmentation. IEEE Transactions on Pattern Analysis and Machine Intelligence, 22(8), 888-905.

Tang, J. (2010, April 16-18). A color image segmentation algorithm based on region growing. In $20102 n d$ International Conference on Computer Engineering and Technology (Vol. 6, pp. V6-634). Chengdu, China.

Tang, R., Fong, S., Yang, X. S., \& Deb, S. (2012, August 22-24). Integrating nature-inspired optimization algorithms to K-means clustering. In Seventh International Conference on Digital Information Management (ICDIM 2012) (pp. 116-123). Macau, China. 
Thennarasu, S. R., Selvam, M., \& Srihari, K. (2020). A new whale optimizer for workflow scheduling in cloud computing environment. Journal of Ambient Intelligence and Humanized Computing, 11(5), 1-8.

Yang, X. S. (2010). A new metaheuristic bat-inspired algorithm. In Nature inspired cooperative strategies for optimization (NICSO 2010) (pp. 65-74). Heidelberg, Germany: Springer.

Ye, Q., Gao, W., \& Zeng, W. (2003, July 6-9). Color image segmentation using density-based clustering. In 2003 International Conference on Multimedia and Expo. ICME'03. Proceedings (Cat. No. 03TH8698) (Vol. 2, pp. II-401). Baltimore, MD, USA.

Zaitoun, N. M., \& Aqel, M. J. (2015). Survey on image segmentation techniques. Procedia Computer Science, $65,797-806$.

Zhang, Y., Huang, D., Ji, M., \& Xie, F. (2011). Image segmentation using PSO and PCM with Mahalanobis distance. Expert Systems with Applications, 38(7), 9036-9040. 
